\title{
Review of: "The obesity-linked human IncRNA AATBC regulates adipocyte plasticity by stimulating mitochondrial dynamics and respiration"
}

\author{
Didier Pisani
}

Potential competing interests: The author(s) declared that no potential competing interests exist.

The proposed paper by Giroud and collaborators is a highly interesting study demonstrating the role of the human specific AATBC IncRNA in adipocyte biology and its potential link with obesity. This opens new avenues in molecular comprehension of thermogenic activity in human. Despite the difficulty to study IncRNA in human situation, the authors have developed a large part of the available approaches to characterized and deeply analysed the role of a AATBC in human model.

First, they developed a robust crossed RNAseq approach to identify new IncRNAs potentially involved in human thermogenic activity. This allowed the identification of several candidates among them AATBC retained their attention.

Using relevant human cell models, the authors validated their target and demonstrated the correlation between AATBC expression and thermogenic adipocyte differentiation and then, by knock-down or overexpression strategies, its implication in the acquisition of late thermogenic phenotype and mitochondrial dynamic.

Then, the authors overexpressed this human specific IncRNA in mice. Only moderated results on leptin expression and TG level were found, certainly demonstrating the difficulty to study human specific IncRNA in rodent models.

Finally, the authors studied AATBC expression in several cohorts of people with various BMI and identify a link between AATBC expression and several parameters of obesity.

This work will be of high interest for several scientific fields from molecular biology of IncRNA to pathophysiology of obesity. Without discuss about the form and presentation of the paper, I'm convinced that it can be improved by adding some additional results, first to deeply define the impact of AATBC on adipocyte biology and then to clarify/improve some already displayed experiments.

1/ As a general comment, despite robust correlation between AATBC expression and thermogenic phenotype of human adipocyte, the authors failed to uncover a clear link between AATBC and modulation of the thermogenic activity. Regulation of this activity is a complex program involving several different pathways, giving difficult its deciphering. Nevertheless, the authors did not investigate the lipolysis pathway which is crucial in activation and activity of adipocyte thermogenic capacities. Moreover, several points displayed in this work can indicate the central role of lipolysis in the modulation of thermogenic 
activity by AATBC, along these:

- the approaches used for the RNAseq and the in vitro validation have been performed using NE and forskolin stimulated adipocytes, two known lipolysis inducers. This could have influenced the results toward "lipolysis affected IncRNAs" in addition to "thermogenic linked IncRNAs".

- the results obtained in human adipocyte cell model could involved lipolysis, as Ucp1 expression, respiratory activity, and mitochondrial dynamic are modulated.

- the results obtained in vivo in mice with the variation of TG and leptin circulating levels can reflect browning associated with increased lipolysis.

In conclusion, this study will merit to integrate a deep analysis of lipolysis pathway in all the in vitro and in vivo models used. Glycerol release as well as TG content, phosphorylation of lipases and perilipins, and lipid droplet size analysis may be envisaged in this way. Ambiguously, authors discuss about lipolysis and AATBC without displaying any result.

2/ More specifically, some experiments should be added to clarify several results already displayed in this work:

- AATBC is only validated using the models used for the RNAseq. Despite the high relevance of these models, it will be very interesting to validate AATBC expression it in other available models as SGBS or clonal human brown adipocyte cell lines.

- Seahorse experiments are confusing as they were not realized following the same protocols and did not display ECAR results. These should be homogenised. The sequence "oligmycin A then isoproterenol" will allow to analyse the uncoupling activity "independently" and "due to" isoproterenol stimulation, an important point if AATBC alone is sufficient to modulate a pathway activating Ucpl activity. Finally, as basal OCR are differents between conditions, analyse of uncoupling should be performed additionally as $\%$ of basal, an approach which can uncover interesting results.

- The study of mitochondrial dynamic is interesting but may be improved by adding analysis of DRP1 phosphorylation, a central actor of adipocyte thermogenic activity.

- Histologic analysis of injected tissues in mice must be displayed as they will be certainly informative about at least lipolytic and thermogenic phenotype of the tissues.

- Presentation of the cohorts should be clarified; the present form is confusing.

- In the first analysed cohort, the absence of men with $\mathrm{BMI}<30$ severely limits interpretation of the results. This part of the analysis could be excluded.

- Discussion could be improved by adding a more general integration of the results with scientific knowledges.

Thank a lot for this interesting study and I hope that these comments will be helpfull for you. 
